\title{
Fluorescence excitation on tapered polymer optical fibers through microfiber evanescent field
}

\author{
E. Rodríguez-Schwendtner ${ }^{a}$, M.C. Navarrete ${ }^{a}$, Ó. Esteban $^{* b}$, N. Díaz-Herrera ${ }^{c}$, A. González-Cano $^{\mathrm{c}}$ \\ ${ }^{a}$ Applied Optics Complutense Group/Physics Faculty, Universidad Complutense, 28040 Madrid, Spain; ${ }^{b}$ Photonics \\ Engineering Group/Dept. of Electronics, University of Alcala, 28871 Alcalá de Henares, Spain; ${ }^{\mathrm{C} A p p l i e d ~ O p t i c s ~}$ \\ Complutense Group/Optics and Optometry Faculty, Universidad Complutense, Arcos de Jalón 118, 28037 Madrid, Spain
}

\begin{abstract}
In this work, we show the feasibility of using the evanescent field of a microfiber to excite the fluorescence of a fluorophore deposited on the surface of a polymer optical fiber. The fluorescence intensity gathered by the polymer optical fiber from the emitted on its surface has been measured for several gaps with the excitation fiber, showing a remarkable influence of the evanescent field at distances as high as $2.5 \mathrm{~mm}$. A further development of a device based on wrapping the microfiber around the polymer one has been measured, proving a more efficient fluorescence excitation.
\end{abstract}

Keywords: Fluorescence, polymer optical fiber, microfiber.

\section{INTRODUCTION}

Among the different available optical fiber sensors, those based on polymer optical fibers (POF) have recently attracted particular attention $[1,2]$. These fibers present the same advantages of conventional ones, together with larger diameter; easy handling including cut and polishing procedures; durability, flexibility and relative low cost [3]. These special features make them specialty suitable for their use in continuous environmental monitoring where budget is a fundamental issue [4], as well as their biocompatibility give advantage to their use in medical field [3] where rough handling and disposability are the preferred operating way.

Although medical and environmental monitoring can use different techniques, the main option for bio/chem sensing is fluorescent measurement [3,5]. Fluorescence-based POF sensors can be easily found in literature [6,7] although their efficiency can be compromised, depending upon the light coupling procedure. In this sense, the use of side-illumination technique provides a promising light coupling method to improve efficiency while keeping a relatively simple setup $[2,8]$. Besides, it is possible to get strong evanescent fields with recently developed devices based on optical fiber tapering; the so called micro/nano fibers (MNF), where a uniform waist region has a size comparable to the wavelength of the propagating light [9].

In this work, we present a combined use of MNF and POF to get a high efficient fluorescence signal taking advantage of the strong evanescent field provided by the MNF. This field excites a luminophore deposited on a POF which also act as gathering fiber [10]. With the proposed setup it is possible to avoid the use of both bifurcated fibers and block filters needed to get the fluorescence signal without cross-interference of the excitation one. Furthermore, since the excitation light is guided by an optical fiber, it opens the way to launch multiple wavelengths towards the fluorescent area for exciting several fluorophores simultaneously or in different locations along the POF.

*oscar@depeca.uah.es; phone 34 918856691; fax 34 918856591; http://grifo.depeca.uah.es/

24th International Conference on Optical Fibre Sensors, edited by Hypolito José Kalinowski, José Luís Fabris, Wojtek J. Bock, Proc. of SPIE Vol. 9634, 96346D · C 2015 SPIE CCC code: $0277-786 \mathrm{X} / 15 / \$ 18 \cdot$ doi: $10.1117 / 12.2193920$ 


\section{TRANSDUCER FABRICATION AND EXPERIMENTAL SETUP}

The proposed device includes two tapered optical fibers. The first one is a standard optical fiber (SMF-28) which is used to excite the fluorescence. This fiber is tapered following the well-known travelling-burner technique, where the silica is heated in an oscillatory way around the length to be stretched while is gently drawn in opposite directions. With this procedure we have fabricated tapered single-mode fibers from standard silica fibers optimized for transmission in the communications C-band $(1.55 \mu \mathrm{m})$. The parameters of the obtained tapers are: $15 \mu \mathrm{m}$ waist diameter (which represents a tapering ratio of 8.33), $10 \mathrm{~mm}$ waist length for a total taper length of about $52 \mathrm{~mm}$, and transmision losses around 1.5 dB.

The second tapered fiber is a made in a PMMA fiber with an outer diameter of $1 \mathrm{~mm}$, which is reduced to $850 \mu \mathrm{m}$ by chemical etching. In this case, the procedure we have followed is the immersion of the unjacketed fiber in pure acetone until the final diameter of the taper waist is below the core diameter. The actual diameter is measured at regular time intervals by optical and mechanical methods until the taper waist is around $850 \mu \mathrm{m}$. The selection of this diameter allows us to fulfil the compromise between light collection, available surface for the fluorophore deposit and mechanical strength of the transducer. Although this figure implies the removal of a superficial layer of the core, the transmittance of the fiber is not compromised since the outer medium still has a lower refractive index than the core. A final taper length of about $70 \mathrm{~mm}$ is obtained with this method. Afterwards, a water-based fluorescent ink with high quantum yield is deposited on the surface of this POF tapered fiber to generate the fluorescence transducer.

The evaluation of the fluorescence coupling inside the polymer optical fiber by means of the fluorophore excitation due to the light leaking from the microfiber, that is used as a light source; is considered. The setup used is schematically depicted in figure 1. A fixed platform is placed in front of another one mounted on a linear translation stage. This optomechanical arrangement permits either to bring the MNF close to the POF or away from it. A Thorlabs $405 \mathrm{~nm}$ laser diode (CPS405) is focused in the MNF. The power coupled into this fiber is about $0.6 \mathrm{~mW}$. Then, the fluorescence excited on the POF surface is coupled into this fiber, which is connected to a CCD spectrometer and a laptop where the gathered spectrum can be recorder for further analysis. This setup is enclosed in a dark container, keeping outside both the laser diode and the laptop just to prevent any other light source interference in fluorescence generation.

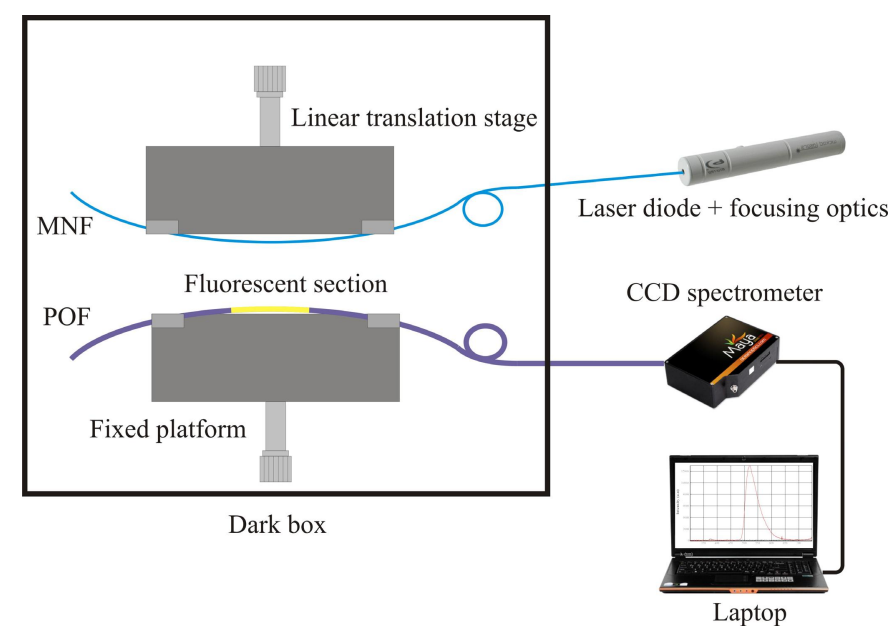

Figure 1. Scheme of the used setup for fluorescence measurements. Both excitation fiber (MNF) and fluorescent fiber (POF) are kept inside a dark box to prevent fluorescence generation from laptop screen and laser stray light.

\section{EXPERIMENTAL RESULTS}

With the setup depicted in the previous section, the intensity of the fluorescence signal coupled to the POF has been measured as a function of gap between POF and MNF. When the POF is fixed by clamps, there exists a slight residual curvature due to the fabrication procedure. Anyway, both fibers, POF and MNF run almost parallel along the complete interaction length. 
Then, the MNF can be moved either towards or away from the POF fiber in a controlled way from close contact to a distance limited by the signal gathering by the spectrometer. In our case, the gap between fibers has been changed in 0.2 $\mathrm{mm}$ steps from 0 to $10 \mathrm{~mm}$.

Once fixed the relative distance between the fibers, the fluorescence signal is recorded in the CCD spectrometer with an integration time of $3 \mathrm{~s}$. In figure $2 \mathrm{a}$ the obtained spectra for several distances are plotted, namely both fibers in contact ( 0 $\mathrm{mm}$ ), and for gaps of $2.2 \mathrm{~mm}, 4.2 \mathrm{~mm}$ and $9.48 \mathrm{~mm}$. In figure $2 \mathrm{~b}$, the evolution of the spectra maxima are also plotted as a distance function. As can be seen, this figure confirms the expected exponential decay behavior of the evanescent field of the guided modes by the microfiber, together with the increase in fluorescence signal as the excitation source becomes more intense as predicted by Stern-Volmer equation [2]. Nevertheless, a remarkably high relative distance between MNF and POF still renders a signal intense enough at a distance of around $2.5 \mathrm{~mm}$, which opens the way to include any media inside the gap between the fibers.
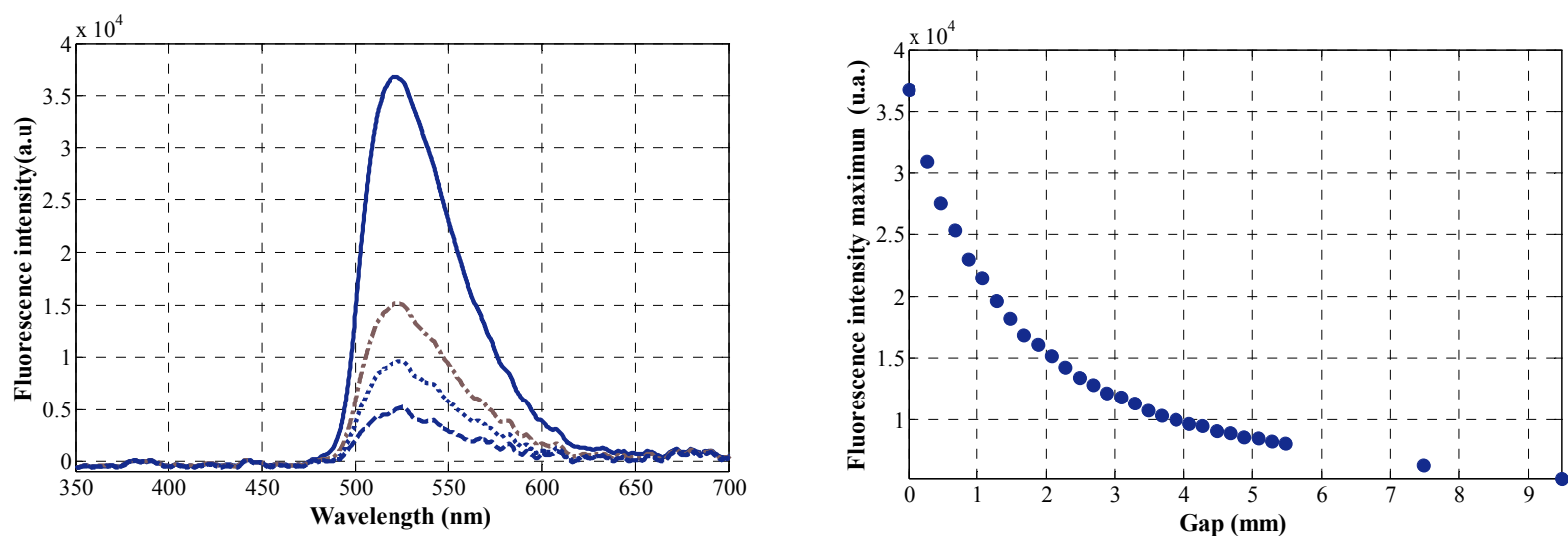

Figure 2. a) Fluorescence spectra measured at different distances between MNF and fluorescent POF: 0mm (solid line), $2.2 \mathrm{~mm}$ (dash-dotted line), $4.2 \mathrm{~mm}$ (dotted line) and $9.4 \mathrm{~mm}$ (dashed line). All spectra have been measured with $3 \mathrm{~s}$ integration time. As can be seen, the $405 \mathrm{~nm}$ excitation wavelength does not appear. b) Maximum value of the measured spectrum for each tested gap between fibers. The exponential decay can be clearly identified.

From these results we proved the feasibility of exciting fluorescence on the surface of a tapered polymer fiber through the evanescent wave of the light guided by a micro fiber, even with a MNF diameter as large as $15 \mu \mathrm{m}$. As the signal becomes more intense when the MNF is closer to the POF, we have also developed a new device in which the MNF is wrapped around the fluorescent POF fiber as depicted in figure 3a. The microfiber is twice turned around the tapered section of the POF with the fluorescent ink deposited on it. Following the already depicted procedure to measure the fluorescence signal, the integration time had to be reduced to the milliseconds range in order to prevent the CCD saturation. The obtained results are plotted in figure $3 \mathrm{~b}$, where the recorded signal for $250 \mathrm{~ms}$ (solid line) and $100 \mathrm{~ms}$ (dashed line) are shown.
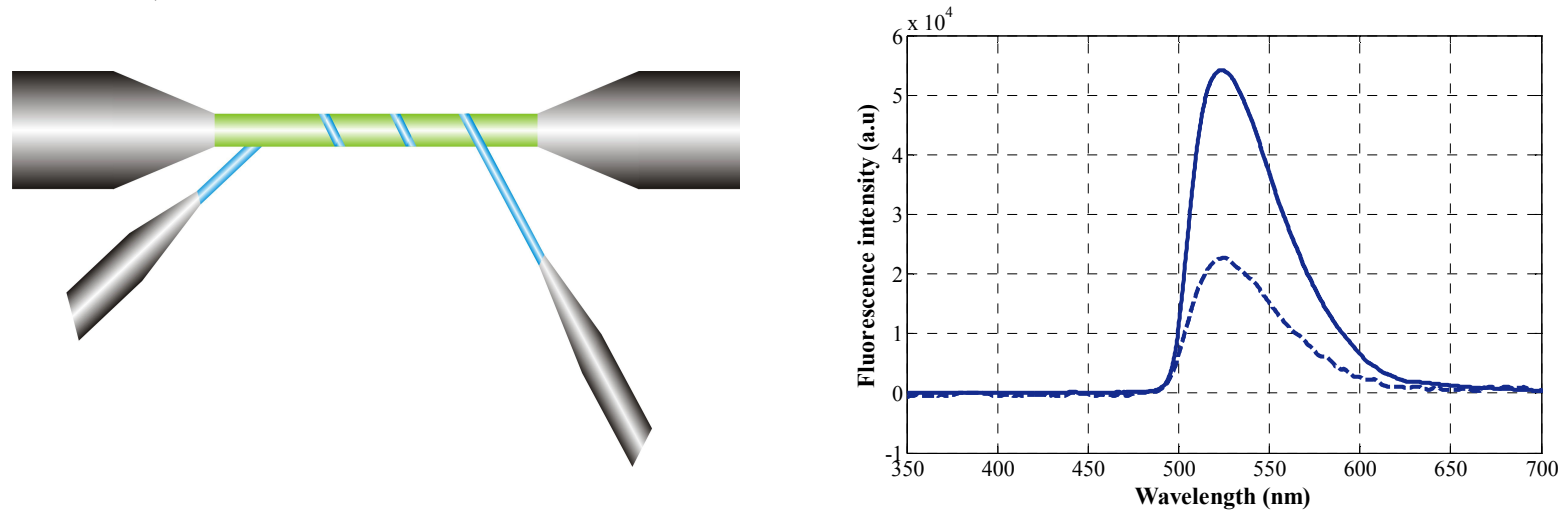

Figure 3. a) Developed device with a microfiber wrapped around a polymer optical one. b) Measured fluorescence spectra with the MNF coiled twice around POF for $250 \mathrm{~ms}$ (solid line) and $100 \mathrm{~ms}$ (dashed line) integration time. 
As can be seen, with this device, the signal level is of the same magnitude order than the obtained with the previous measurements when the fibers are close to each other, but with a significant reduction of the time needed to get this value. This could lead to more compact and faster fluorescence-based sensors devices, with multiple discrete excitation wavelengths in a single light source, namely the MNF.

\section{CONCLUSIONS}

In this work we have shown the feasibility of the use of microfibers as light source for exciting the fluorescence of a luminophore deposited on the surface of a polymer optical fiber. The dependence with the distance between the micro fiber and the polymer one has been measured, leading to the development of a hybrid device where the MNF is wrapped around the POF. The characterization of this later device shows how the coupling efficiency increases gathering the same fluorescence signal level with a much lower integration time. A further advantage of this device would be the possibility of launching several laser wavelengths into the same fiber which would act as light source to excite several fluorophores locates at the same POF section simultaneously, leading to multiparameter measurements with a single device.

\section{ACKNOWLEDGMENTS}

This work has been partially supported by the European Project ECOAL-MGT (Ecological Management of Coal Piles in Combustion), ref. ECOAL-MGT-SOE-P2-P714, and by the Comunidad de Madrid program SINFOTON (Sensores e Instrumentación en Tecnologías Fotónicas), ref. S2013/MIT-2790.

\section{REFERENCES}

[1] J. Zubia, J. Arrue, 'Plastic Optical Fibers: An Introduction to Their Technological Processes and Applications', Optical Fiber Technology 7, 101-140 (2001)

[2] C. Pulido, Ó. Esteban, 'Improved fluorescence signal with tapered polymer optical fibers under sideillumination', Sensors and Actuators B 146, 190-194 (2010)

[3] R.J. Bartlett, R. Philip-Chandy, P. Eldridge, D.F. Merchant, R. Morgan, P.J. Scully, 'Plastic optical fibre sensors and devices', Transactions of the Institute of Measurement and Control 22 (5), 431-457 (2000)

[4] L. Bilro, N. Alberto, J. L. Pinto, R. Nogueira, 'Optical Sensors Based on Plastic Fibers', Sensors 12, 1218412207 (2012)

[5] Yinquan Yuan, Liyun Ding, ' Theoretical investigation for excitation light and fluorescence signal of fiber optical sensor using tapered fiber tip', Optics Express 19, 21515-21523 (2011)

[6] C. Pulido, Ó. Esteban, 'Tapered polymer optical fiber oxygen sensor based on fluorescence-quenching of an embedded fluorophore', Sensors and Actuators B 184, 64-69 (2013)

[7] D. Haigh-Flórez, C. de la Hera, E. Costas, G. Orellana, 'Microalgae dual-head biosensors for selective detection of herbicides with fiber-optic luminescent $\mathrm{O}_{2}$ transduction', Sensors and Actuators B 54, 484-491 (2014)

[8] C.O. Egalon, 'Side illuminated multi point multi parameter optical fiber sensor', World Patent WO/2010/0088591, 4 Nov. 2010

[9] G. Y. Chen, M. Ding, T. P. Newson, G. Brambilla, 'A Review of Microfiber and Nanofiber Based Optical Sensors', The Open Optics Journal 7, 32-57 (2013)

[10] C. Pulido, Ó. Esteban, 'Multiple fluorescence sensing with side-pumped tapered polymer fiber', Sensors and Actuators B 157, 560- 564 (2011) 